\title{
Virtual Reality Becomes Reality in Plastic Surgery
}

\author{
CHANTAL L. RAWN, B.S., PAUL J. GORMAN, M.D., \\ WILLIAM P. GRAHAM, III, M.D., THOMAS M. KRUMMEL, M.D., and \\ DONALD R. MACKAY, M.D.
}

ABSTRACT With the new millennium upon us, the increasing use of computers and the Internet are forcing us to evaluate our traditional methods of surgical training and practice. The conventional surgical education method of "see one, do one, teach one" does not sufficiently address the need to achieve standardized, reproducible, and safe teaching methods. Current constraints imposed by managed care require cost-effective and efficient means to educate and evaluate future surgeons. Advances in visualization, instrumentation, and computing power have led to developments in virtual reality (VR) and simulation. The advent and growth of this technology offers an effective means of addressing concerns regarding plastic surgical training, education, and practice in the modern healthcare environment.

Keywords Virtual reality, simulation, computer-assisted surgery, plastic surgery training, preoperative planning

The current surgical teaching method is based upon the preceptor or apprenticeship model, in which the resident surgeon learns with small groups of peers and superiors, over time, in the course of patient care. Surgeons have always acquired most of their operative and judgment skills through "learning by doing." Though an essential portion of surgical practice, the majority of tech-

C.L.R. Researcher; P.J.G., Post-Doctoral Research Fellow, Department of Surgery, Stanford University School of Medicine, Stanford, CA; W.P.G., Professor of Surgery and Anatomy, Division of Plastic Surgery, The Hershey Medical Center, Penn State University College of Medicine, Hershey, PA; T.M.K., Chairman, Department of Surgery; Professor, Department of Surgery, Stanford University School of Medicine, Stanford, CA; D.R.M., Associate Professor of Surgery and Pediatrics; Chief, Division of Plastic Surgery, The Hershey Medical Center, Penn State University College of Medicine.

Copyright ( 2000 by Thieme Medical Publishers, Inc., 333 Seventh Avenue, New York, NY 10001, USA. Tel.: +1(212) 584-4662. 0892-3957,p;2000,14,2,105,118,ftx,en;pps00046X. 
nical skill instruction occurs through fairly unstructured operating room exposure. Ideally, exposure to operative practice should commence at an early training level and in an organized fashion that allows the breakdown of tasks into simple steps ${ }^{2}$ in a-time flexible environment. ${ }^{3}$ These prerequisites cannot be fulfilled in today's operating rooms. Even if this were possible, accurate assessment of the resident's progress would pose another significant challenge.

Due to the nature of surgical practice, the approach described above will likely remain a cornerstone of the surgical education process. Studies analyzing learning style preferences of surgical residents show that most favor a problem-solving and hands-on approach, ${ }^{4,5}$ which may partly explain why this form of teaching has been so successful. The "learning by doing" approach, though, fails to provide skill acquisition in an organized fashion. Teaching opportunities are dependent upon the random flow of patients through the office, clinic, emergency unit, and operating room. The operating room itself provides a venue to demonstrate technique and place the operation in the context of overall patient management. Indeed, the OR has been termed "the surgeon's classroom and laboratory extraordinaire."6 However, the variability in patient flow results in significant unpredictability in the educational content provided to the trainees, and precludes any organized curriculum.

Changes in healthcare financing have placed enormous pressure on medical training, including that of plastic surgeons. ${ }^{7}$ How do we manage the transition from our current system to one that is efficient, outcomes-based and cost-effective? The purpose of this paper is to explore the use of virtual reality, simulation, and other computer-assisted techniques in plastic surgery education, training, and practice. In order to more clearly understand these issues, we will address the following topics: the concepts of virtual reality and simulation and an overview of the development and current state of computer-assisted techniques in plastic surgery.

\section{VIRTUAL REALITY}

Virtual reality (VR) offers a three-dimensional computer-generated environment that, if successful, mimics the real world. The term "virtual reality" was coined by Jaron Lanier, founder of VPL Research, in the late 1980s. Since then, VR has also been defined as a human-computer interface that simulates realistic or imaginary environments while immersing the participant in a three-dimensional interactive world-“cyberspace."

The basic elements of a virtual reality system in medicine are: an appropriate computer plus software, a graphics card and display monitor, an image database and physical interface device. The software program controls the object and its interaction in the virtual world while the hardware performs the calculations necessary to update the changing images. Raw data for the image database are obtained from one or several of the following techniques: two-dimensional photographs and radiographs, three-dimensional computed 
tomography (CT), magnetic resonance (MR) and ultrasound images. MRI and ultrasound are preferred in some cases because these techniques avoid irradiating the patient ${ }^{11,12}$ and, particularly in MRI, display soft tissue with greater accuracy. ${ }^{11}$

Once the images have been obtained, they must be integrated, rendered, and animated using the software. To successfully create a virtual environment training system, the following issues must be considered: resolution, update rate, realism, and lag time. In the virtual world, objects are created from a patchwork of flat two-dimensional shapes known as polygons. The more polygons used to render the object, the greater the precision and detail. To improve the realism of the object, polygons are colored and shaded during the volume rendering process. ${ }^{8,13}$ High-resolution medical data result in a huge number of polygons. To limit the hardware processing time, or latency, additional software is used to reduce the total number of polygons and form a geometric approximation of the object. ${ }^{14,15}$ Therein lies the trade-off in virtual medical environments. Either the image resolution must be sacrificed or the virtual interaction is not in real-time. Real-time refers to the ideal frame refresh rate of 24 to 30 frames per second, at which the eye does not distinguish from one frame to the next.10,14,16,17 Recent advancements in graphics card technology have allowed for this superior polygon per second calculation to be realized.

\section{SIMULATION}

Virtual reality provides the computer model (representation) to create immersion and interaction with a lifelike, multidimensional, simulated environment. Simulation, loosely construed, is the act of assuming the outward qualities or appearances of a given object(s) or process or series of processes. To achieve this simulation, three elements of VR technology are essential: immersion, haptic sensibility, and a navigational capability. By immersion one experiences his/her presence in a virtual world provided by visual, auditory, and tactile perceptions of computer-generated environmental stimuli. Vision is the most powerful factor in entering the virtual environment. Once the imaging issues have been resolved, there are multiple interfaces with which to display the visual data, all of which depend on the level of immersion desired.

Ivan Sutherland's work on interactive head-mounted displays in the mid 1960s initiated the development of 3-D graphical visualization, though it was not until the mid-1980s that evolving components allowed Lanier and others to develop viable head-mounted displays (HMDs). ${ }^{18}$ Most HMDs offer the illusion of total immersion into a virtual world by providing visual information while simultaneously blocking visual contact with the outside world. ${ }^{8,10}$ In some instances, a partial visual immersion creating an augmented, rather than virtual, reality is necessary to provide the surgeon with immediate access to the "real" world. ${ }^{19}$ In this case, a see-through HMD is used in conjunction with video tomography to superimpose images on the real patient. 
Another alternative to the HMD are the binocular omni-orientated monitor (BOOM) displays in which a high-resolution cathode ray tube (CRT) is projected on a mounted counterbalanced arm with 6 degrees of freedom. ${ }^{8}$ This system allows for immediate return to the real environment when necessary. Similarly, heads-up displays are glass screens positioned in front of the observer that allow for the overlay of virtual images on the actual view. ${ }^{8}$ In addition to visual cues, auditory and other sensory cues can be added to enhance the overall sense of immersion.

Navigation permits exploration, physical movement, and interaction within the environment. Tracking devices are essential to creating this sense of travel through the virtual world. Motion-coupled HMDs or motiontracking body suits monitor the position of the participant in relation to a fixed point using electromagnetic, mechanical, and infrared sensors. Physical interfaces include wired gloves, wands, force balls, mice, biosensors and voice recognition modules to enable the user to perform actions in 3-D space with varying degrees of freedom (the maximum is a 6-degree of freedom device with $\mathrm{X}, \mathrm{Y}, \mathrm{Z}$, pitch, roll, and YAW).$^{8}$

The user's ability to manipulate objects and gain haptic information is the final and most complex step in creating a simulation environment. Haptic capability includes tactile feedback (the sensation of surface features, texture, and slip), force feedback (resistance or reciprocal force) and kinesthesia (awareness of orientation and position in space). ${ }^{20}$ These can be provided through tactile and force feedback devices that are currently in varying stages of experimental and commercial development. ${ }^{20,21}$ Essentially, force is exerted on the operator based on motion and interaction with the virtual model. One of the factors being assessed is the time lag between the participants' movements within the VR world and the system's response. In order to sustain the illusion of reality it must be less than $100 \mathrm{~ms} .{ }^{16}$ Studies have indicated that at a higher latency, the user will over- or under-compensate and potentially experience motion sickness. ${ }^{10,22}$

\section{INDUSTRIAL APPLICATIONS}

Virtual reality (VR) and its use in simulation has caught the attention of the media and public alike as an exciting new technology with multiple applications. It has been used in a variety of educational, training, testing, analysis, research and development, and entertainment settings. ${ }^{23,24}$ The highly visual and interactive nature of VR has proven to be useful in understanding complex 3D structures and for training in visual-spatial tasks. ${ }^{25}$ Simulation affords effective learning experiences in groups or alone, can suit learners of varying backgrounds, is interactive and fun, and is compelling because one can see the consequences of one's actions. ${ }^{26}$ 
VR is perhaps most well known for its role in civilian and military pilot and astronaut training. A simulated VR environment provides the ideal opportunity for pilots and astronauts to safely practice dangerous or difficult maneuvers in certain environments that cannot be replicated without VR. ${ }^{10}$ Simulation also provides a standardized means of training and re-certifying for the military and aviation industry. ${ }^{27}$ In fact, in 1955 the Federal Aviation Administration required that all pilots pass an annual skills performance test in a simulator for re-licensing. ${ }^{28}$

In addition to air and space flight training, training simulators for military and commercial vehicles, mechanical system maintenance, and nuclear power plant operation exist. Transport companies use simulators to prototype and test ground and air transport vehicles, primarily because they provide testing environments that are controllable, secure, and safe. The cost effective use of simulators as described has demonstrated the utility of realtime simulation as a training tool, and has sparked interest in the development of simulators for other potentially dangerous environments (i.e., new or complex medical procedures). ${ }^{24}$

Simulation and VR in medicine has been undertaken in a variety of settings. Paramedics are taught triage and assessment skills with this technique, and Advanced Trauma Life Support (ATLS) and Advanced Cardiac Life Support (ACLS) courses rely upon simulated scenarios to teach and test skills. Screen and mannequin-based simulators have been used in anesthesia training to ensure that clinicians will be exposed to atypical and stressful situations. ${ }^{29,30}$ Efforts to show that these simulators improve clinical performance have been ambiguous. While Chopra and others showed that anesthesiologists trained on a "high fidelity anesthesia simulator" responded more quickly and appropriately when handling crises on the simulator, controlled validation studies involving human patients have not been carried out due to an unacceptable risk level. ${ }^{31}$

The ability of simulators to prepare individuals for potentially lifethreatening situations in dynamic, unpredictable environments demands their use in surgery. Early developments in the surgical field included the virtual abdomen created by Satava and Lanier and the hip arthroplasty planning application by Rosen et al. ${ }^{32,33}$ Applications ranging from virtual endoscopy, to interactive anatomy teaching modules, to preoperative planning in neurosurgery and prostate cancer surgery have been described. ${ }^{16,34-37}$ A series of dedicated conferences have sparked interest in this field, and reports on VR applications in surgery can be found in the medical, computer science, engineering, and popular lay literature.

\section{PLASTIC SURGERY APPLICATIONS}

Perhaps one of the greatest contributions of VR and simulation technology is to the field of plastic surgery. Applications to plastic surgery can be subdi- 
vided as follows: training and education, surgical planning, intraoperative guidance, and telesurgery.

\section{TRAINING AND EDUCATION}

The present situation in surgical education necessitates an alternative method for training plastic surgeons in the future. Plastic surgery often involves complex and sensitive anatomic structures. Virtual environments provide the opportunity to view and interact with these complicated configurations without the restrictions of cadavers or models. ${ }^{13,38}$ Endoscopic sinus surgery (ESS), in which the surgeon is expected to navigate complex labyrinthine passageways while bypassing the eye socket and internal carotid artery, though not strictly within the realm of plastic surgery, presents one such example. Currently, students are taught by dissecting a cadaver and memorizing anatomy from two-dimensional images. Unfortunately, cadaveric material is expensive, is in limited supply, and does not demonstrate the subtle tissue characteristics of real tissue. Moreover, it is unlikely that rare anatomic variations will be well represented in cadavers.

Advances in tissue modeling, graphics, and haptic instrumentation have enabled Wiet et al. to construct an ESS simulator to assist in the education and training of this procedure. ${ }^{13}$ Similar work has been done by Ecke et al. in the creation of a nasal endoscopy simulator (NES) with up to date tracking sensors and acoustic stimuli. ${ }^{14}$ The simulated nasal environment enables trainees to perform sinus surgery in a safe, controlled, and reproducible fashion. Additionally, rare and unusual anatomic patient simulations can be experienced, thereby eliminating the waiting associated with random occurrences and chance opportunity to do these procedures. ${ }^{36}$ The cost and dangers in use of cadaveric and animal material are also bypassed. ${ }^{39}$

Simulation provides advantages that are useful in skill development and in skill assessment and maintenance. Currently, surgical competence is characterized by vague terms such as "too long, too short" or "too close, too far." Virtual reality, in combination with the principles of fuzzy logic, provides an ideal method of quantitatively assessing surgical competence. ${ }^{40}$ VR trainers also allow residents and experienced surgeons to return to the same procedure multiple times as a refresher. The flexibility afforded by round-the-clock access to the simulator is convenient and provides an efficient learning environment for hectic schedules.

Initial validation studies using simulators have shown differences between experienced and novice surgeons, that training scores improve over time, and that simulator task performance is correlated to actual task performance. ${ }^{41-43}$ While simulators cannot replace the learning experience of the real operating room, they can prepare the trainee for the performance of his/her first real procedure under supervision without risk to the trainee or the "patient." 


\section{SURGICAL PLANNING}

VR technology serves as a useful adjunct to traditional plastic surgery planning techniques. Reconstructive and cosmetic surgeries require extensive preoperative planning and a clear idea of the desired results. In some cases, even a slight deviation of a few millimeters from the desired repositioning or reassembling can result in an undesirable outcome. Early computer imaging techniques were completed by Papel et al. to facilitate preoperative analysis and postoperative projections in facial plastic surgery. ${ }^{4}$ Using what is called an expert system, the surgeon was able to opt for several different actions based on predetermined histories and images, thereby establishing the ideal operation for the particular operation. ${ }^{45}$

Recent research in image processing and segmentation of CT and MRI scans has enabled reliable patient-specific 3D reconstructions of important anatomic structures. $46-48$ In some cases $3-\mathrm{D}$ imaging data has been used in stereolithographic skull and facial modeling to further simulate complex anatomical relationships. ${ }^{49-53}$ While this technique provides actual size models with tactile and 3D stimuli, it does not predict soft tissue shifting, overall aesthetics, or allow for repeatable manipulation and interaction. Virtual reality simulation, on the other hand, provides for all of these modalities.

In craniofacial surgery, virtual reality has been used to simulate skeletal deformities using deformable tissue modeling to estimate soft tissue changes preoperatively. $15,38,39,54,55$ Patient-specific 3 -D images can be rotated to visualize contours of the craniofacial deformity and allow the surgeon to "try out" several different reconstructions with visual and tactile feedback. ${ }^{39,45}$

The ability to preoperatively simulate a complicated reconstruction enables the surgeon to predict with improved accuracy the outcome. This is especially difficult in the simulation of postoperative facial expressions in facial palsy patients because of the technological challenges associated with soft tissue deformation. However, progress has been made in this arena, as demonstrated by Tanak et al. and Fujuino and have enormous implications for improved patient outcome. ${ }^{53,56}$ Work is also in progress to simulate airflow in nasal surgery planning with the Airflow Simulation Tool for Human Medical Application (ASTHMA), with mixed results. ${ }^{57}$ It is clear that plastic surgery is in the process of employing virtual reality and simulation as preoperative tools for maximizing patient functionality and overall aesthetics.

\section{COMPUTER-AIDED SURGERY}

The integration of advanced imaging technology, image processing and 3-D graphical capabilities have led to great interest in image-guided and computer-aided surgery. Navigation in surgery relies upon stereostatic principles, based on the ability to locate a given point using geometric references. ${ }^{58}$ 
The various virtual reality devices previously described allow for multiple intraoperative applications of the technology. Korves et al. has created the Aachen Computer-Assisted Surgery System that allows every step of the surgical procedure to be simulated ahead of time on a screen using the "LookAhead" function. ${ }^{38}$ The system has been used in 407 paranasal sinus procedures and is particular useful in endoscopic and endonasal procedures because it allows the surgeon to "virtually" cut away at the surface he or she is viewing through the endoscope without actual resection.

The "double-doughnut" or open-magnet MRI machine used at Brigham and Women's Hospital in Boston employs a similar setup. By constantly updating and displaying intraoperative images on a monitor and comparing them to preoperative MRIs, the surgeon is able to assess his or her current position with that of tumors or structures of interest with so-called " $\mathrm{x}$-ray vision." "ll The best results (least amount of error in location) have been realized in systems that assist in precise positioning of replacement bone fragments using graphic and numerical markers on a screen. ${ }^{39,59,60}$ In one example, bone was replaced to the predefined optimum position in multisegment craniofacial surgery using infrared motion tracking. ${ }^{59}$

In order to eliminate the need to look up at a monitor, Edwards and others have created an augmented reality system using an operating microscope for otolaryngology guidance. Utilizing an intraoperative tracking device, 3-D stereo projections appear and are updated in the microscope eyepiece thereby creating a superimposed image on the patient. ${ }^{61}$ Several attempts have been made to take partial immersion one-step further with the advent of seethrough HMDs that superimpose virtual anatomical structures on the operating scene. 19,62 Despite the increased mobility that these devices afford, the image quality is often inferior. ${ }^{19,62}$

Computer-aided surgery also includes the use of robotic systems. An image guided robotic system is being developed by Burghart et al. to provide navigation and support during maxillofacial surgery. ${ }^{63}$ While the technology is currently a work in progress, it offers the potential to integrate autonomous robot assisted actions with a manual human-machine interface. This allows the surgeon to either guide the robot manually or give control to the robotic in areas of complex and sensitive anatomy.

\section{TELESURGERY}

Telesurgery allows the surgeon to operate on a person who is physically separated from him or herself. This is usually done through a master-slave robot, with imaging supplied through video cameras configured to provide a stereoscopic view. The surgeon is reliant upon a $3-\mathrm{D}$ virtual representation of the patient, and benefits from dexterity enhancement afforded by the robotic apparatus. ${ }^{64}$ 
Telesurgery is in its earliest phase of development. A prototype telemanipulator has been used to successfully perform basic vascular and urologic procedures in swine. ${ }^{65,66}$ More advanced systems have been used to perform coronary anastamoses on ex-vivo swine hearts and in humans undergoing endoscopic coronary artery bypass grafting. ${ }^{67-69}$ Currently, some work is also being done to construct a telesimulation system using the Internet. ${ }^{33}$ According to Richard Satava, "By operating on the 'information equivalent' (video image) of the patient using the information equivalent of hand motion (telemanipulation), the field of surgery finally enters the Information Age."70 Advances in computing and Internet capability are necessary before telesurgery is a viable technology in the operating room.

\section{DISCUSSION}

Widespread adoption of virtual reality surgical simulation raises the question of cost, acceptance by the general medical/surgical community, and the limits of technology. The current economics of our healthcare system and managed care demand the best quality of care at the lowest possible price. As a result, training programs need to consider cost-effective methods of providing superior education for the next generation of surgeons. Cost containment in healthcare can be attributed to efficient training (i.e., high quality in the least amount of time), a decrease in the use of expensive resources and operating time, and improved patient outcomes.

While the actual fee of educating one surgeon is not clearly defined, estimates indicate that shortened resident training time as a result of simulators would have a positive impact. ${ }^{40,71}$ Animals, cadaver resources, and stereolithographic modeling can cost more than computer-based training with less flexibility regarding time and place. ${ }^{10,49}$ As the constraints of managed care may lead to standardization and the ability to demonstrate effective techniques and procedures, simulators could provide the ideal tool for standardization and assessment of surgical residents and fellows. ${ }^{40,72}$

The extent to which VR and simulation pervade surgical education and practice will depend upon the advances that are made in computer technology. While the limitations of technology are rapidly decreasing, the fact remains that surgical simulation will not be truly successful without all of the following components: high quality imaging at real-time rates, haptic feedback to provide realistic immersion, and low-cost, easily accessible equipment.

Several ongoing studies in plastic surgery simulation indicated that superior quality real time image refreshment rates are not yet a reality because of the limitations of current computing power. ${ }^{13-15,19,73,74}$ Other reports have shown that collision detection and realistic movement in the virtual environment is currently inadequate. ${ }^{14,54,73,75}$ Graphic anatomical displays that only 
provide a "wet look" until they are prodded, then appear to be covered in a film of plastic do not sufficiently engage the surgeon in a virtual world. In addition, fully immersive simulators require sufficient haptic feedback to create realistic interaction. The study by Edmond et al. notes the challenges in providing the appropriate stretch, retraction and dissection associated with providing realistic soft tissue manipulation in the virtual world. ${ }^{10}$

While the computer technology necessary for optimal surgical simulation is not quite here, prototypes are now available in a more universal and userfriendly environment. Recent technological advances have enabled inexpensive and convenient desktop workstations with high-quality data processing capabilities. This allows surgeons the flexibility to perform their own data processing and navigation. A proof-of-concept study by Freysinger introduced an intraoperative 3-D navigation system that ran on a notebook. ${ }^{76} \mathrm{In}$ the not too distant future, simulation centers and distance learning will be available via the Internet.

\section{CONCLUSION}

While not yet in the mainstream of surgical interaction, the evolving technology of VR and simulation offers the field of plastic surgery revolutionary training, planning and surgical opportunities. The three dimensional virtual world provides users a deeper understanding of spatially complex anatomy. As a tool for learning visual spatial tasks, it provides a direct link between the learner's motor actions and the simulated result. The reduction of risk to patients, the alleviation of costs via the effective use of operating room time, and the ability to perform unlimited procedures on generic models at a time and place convenient to the trainee, are obvious additional benefits. ${ }^{77}$ The value of using this technology as a testing mechanism for certification and recertification, as well as documenting technical progress during training, is clear. Virtual reality and simulation will allow operation prototyping and planning, continued graduate surgical training beyond the residency, and have applications for minimal invasive surgery and robotics.

Changes in healthcare financing increase pressure to provide better clinical outcomes with fewer resources and continually decreasing reimbursement. This development, combined with diminished support for education, threatens the very process by which highly skilled plastic surgeons are produced. ${ }^{78}$ Simulation trainers may offer a valuable part of the solution to this problem. In short, for reasons of educational and procedural quality, safety, and cost, simulation and virtual reality can provide real value now, and its role will almost certainly expand as computer power and availability increase.

What does the future hold for plastic surgery education and training? One key change element in society at large has been the recent explosion in information and connectivity brought on by the Internet. The development of online educational resources in plastic surgery has the potential to increase 
the efficiency of content delivery and provide a common format for educational assessment. ${ }^{78}$ What if the rich educational resources (lectures, conferences, and videos, to name a few) that currently reside in training programs and academic meetings around the country could be captured, edited, and made available for electronic learning in plastic surgery, available anytime, anyplace? What if virtual reality simulators became commonplace online modalities for teaching, planning and performing plastic surgery?

\section{REFERENCES}

1. Folse JR. Surgical education-addressing the challenges of change. Surgery 1996; 120:575-579

2. Barnes RW. Surgical handicraft: teaching and learning surgical skills. Am J Surg $1987 ; 153: 422-427$

3. Griffen WO, Jr. Surgical residency: on-the-job training or education? Am J Surg $1980 ; 140: 720-723$

4. Drew PJ, Cule N, Gough M, et al. Optimal education techniques for basic surgical trainees: lessons from education theory. J Royal Coll Surg Edinburgh 1999;44:55-56

5. Baker JDd, Reines HD, Wallace CT. Learning style analysis in surgical training. Am Surg $1985 ; 51: 494-496$

6. Strodel WE, Zelenock GB. Teaching and learning in the operating room. In: Bartlett RH, Strodel WE, Zelenock GB, Harper ML, Turcotte JG, eds. Medical Education: A Surgical Perspective. Chelsea, MI: Lewis Publishers; 1986:47-51

7. Pearl RM, McAllister H, Pruzansky J. An economic analysis of health care reform and its implications for plastic surgery. Plast Reconstr Surg 1997;99:1-9

8. Burt DE. Virtual reality in anesthesia. Br J Anesth1995;75:472-480

9. Krummel TM. Surgical simulation and virtual reality: the coming revolution [editorial]. Ann Surg 1998;228:635-637

10. Edmond CV, Jr, Wiet GJ, Bolger B. Virtual environments. Surgical simulation in otolaryngology. Otolaryngol Clin North Amer 1998;31:369-381

11. Grimson WE, Kikinis R, Jolesz FA, Black PM. Image-guided surgery. Scientific American 1999;280:62-69

12. Golding $S$, Watt-Smith $S$. Issues in the $3 \mathrm{D}$ imaging-clinical surgery interface. CARS 1999;13:321-325

13. Wiet GJ, Yagel R, Stredney D, et al. A volumetric approach to virtual simulation of functional endoscopic sinus surgery. Stud Health Tech Inform 1997;39:167-179

14. Ecke U, Klimek L, Müller W, Ziegler R, Mann W. Virtual reality: preparation and execution of sinus surgery. Computer Aided Surgery 1998;3:45-50

15. Keeve E, Girod S, Kikinis R, Girod B. Deformable Modeling of Facial Tissue for Craniofacial Surgery Simulation. Computer Aided Surgery 1999;3:228-238

16. Robb RA. VR assisted surgery planning. IEEE Engineering in Medicine and Biology. 1996;March/April:60-69

17. Coleman J, Nduka CC, Darzi A. Virtual reality and laparoscopic surgery [see comments]. Br J Surg 1994;81:1709-1711

18. Schroeder R. Virtual reality in the real world: history, applications and projections. Futures 1993;25:963-972

19. Wagner A, Rasse M, Millesi W, Ewers R. Virtual reality for orthognathic surgery: the augmented reality environment concept. J Oral Maxillofac Surg 1997;55:456-462; discussion $462-463$

20. Chen E, Marcus B. Force Feedback for Surgical Simulation. Proceedings of the IEEE. $1998 ; 86$ 
21. Burdea G, Deshpande S, Popescu V, et al. Computerized hand diagnostic/rehabilitation system using a force feedback glove. Studies in Health Technology and Informatics 1997;39:141-150

22. Kaltenborn KF, Rienhoff O. Virtual reality in medicine. Methods of Information in Medicine. 1993;32:407-417

23. Vince J. Virtual Reality Systems. Reading, MA: Addison-Wesley Publishing Co; 1995

24. Goldiez B. History of networked simulations. Distributed Interactive Simulation Systems for Simulation and Training in the Aerospace Environment. Bellingham, WA: SPIE Optical Engineering Press; 1995:39-58

25. Hoffman H, Vu D. Virtual reality: teaching tool of the twenty-first century? Acad Med 1997;72:1076-1081

26. J Palmer, Synder T. Computer simulations come of age. Experiential and Simulation Techniques for Teching Adults. San Francisco, CA: Jossey-Bass Publishers Inc; 1986:15-24

27. Haber R. Flight simulation. Scient Amer 1986;255:96-103

28. Satava R. Transitioning to the future. J Amer Coll Surg 1998;186:691-692

29. Schwid H. Anesthesia and critical care simulators. Curr Op Anesth 1995;8:532-535

30. Howard S, Gaba D, Fish K, Sarnquist F. Anesthesia crisis resource management training: teaching anesthesiologists to handle critical incidents. Aviat Space Environ Med $1992 ; 63: 763-770$

31. Chopra V, Gesnik B, Jong Jd, Bovill J, Spierdijk J, Brand R. Does training on a anesthesia simulator lead to improvement in performance? Br J Anesth 1994;73:293-297

32. Satava RM. Virtual reality and telepresence for military medicine. Comput Biol Med $1995 ; 25: 229-236$

33. Rosen JM, Soltanian H, Laub DR, Mecinski A, Dean WK. The evolution of virtual reality from surgical training to the development of a simulator for health care delivery. A review. Studies in Health Technology and Informatics 1996;29:89-99

34. Kay CL, Evangelou HA. A review of the technical and clinical aspects of virtual endoscopy. Endoscopy 1996;28:768-775

35. Hoffman H, Irwin A, Ligon R, Murray M, Tohsaku C. Virtual reality-multimedia synthesis: next-generation learning environments for medical education. J Biocommun $1995 ; 22: 2-7$

36. Sinclair M, Peifer J, Haleblian R, Luxenberg M, Green K, Hull D. A Novel Teaching Method for Residents and Pracitioners. Ophthalmology 1995;102:517-521

37. Kelly PJ. Quantitative virtual reality enhances stereotactic neurosurgery. Bull Amer Coll Surg 1995;80:13-20

38. Korves B, Klimek L, Klein H-M, Mosges R. Image- and model-based surgical planning in otolaryngology. J Otolaryngol 1995;24:265-270

39. Mustoe TA, Han H. The effect of new technologies on plastic surgery. Arch Surg 1999;134:1178-1183

40. Ota D, Loftin B, Saito T, Lea R, Keller J. Virtual reality in surgical education. Computers in Biology and Medicine 1995;25:127-137

41. Taffinder N, Sutton C, Fishwick RJ, McManus IC, Darzi A. Validation of virtual reality to teach and assess psychomotor skills in laparoscopic surgery: results from randomized controlled studies using the MIST VR laparoscopic simulator. Studies in Health Technology and Informatics 1998;50:124-130

42. Gorman P, Lieser J, Murray W, Haluck R. Evaluation and skill acquisition using a force feedback, virtual reality-based surgical trainer. Medicine Meets Virtual Reality: Art, Science, and Technology. Amsterdam, the Netherlands: IOS Press; 1999:121-123

43. Weghorst S, Airola C, al. OPe. Validation of the Madigan ESS simulator. Medicine Meets Virtual Reality: Art, Science, and Technology. Amsterdam, the Netherlands: IOS Press; 1998:399-405 
44. Papel ID, Park RI. Computer imaging for instruction in facial plastic surgery in a residency program. Arch Otolaryn Head Neck Surg 1988;114:1454-1460

45. Rosen JM. Advanced surgical technologies for plastic and reconstructive surgery. Otolaryngol Clin North Am 1998;31:357-368

46. Pommert A, Riemer M, Schiemann T, Schubert R, Tiede U, KH H. Three-dimensional imaging in medicine: methods and applications. Computer-Integrated Surgery: Technology and Clinical Applications. Cambridge, MA: MIT Press; 1996:147-154

47. Kikinis R, Gleason L, Jolesz F. Surgical planning using computer-assisted threedimensional reconstructions. Computer-Integrated Surgery: Technology and Clinical Applications. Cambridge, MA: 1996:147-154

48. Ross MD, Montgomery K, Linton S, Cheng R, Smith J. A national center for biocomputation: in search of a patient-specific interactive virtual surgery workbench. Medicine Meets Virtual Reality. Washington, DC: IOS Press and Ohmsha; 1998

49. Millesi W, Rasse M, Eglmeier R, Lindner A, Schobel G, Friede I. Preoperative 3D model planning for reconstruction of the maxilla and the mandible. Computer Aided Radiology 1995:939-944

50. Enislidis G, Schobel GA, Millesi W, Rasse M, Baumann A, Lindner A. Preoperative 3D model planning in secondary correction of established posttraumatic midface deformities. Computer Assisted Radiology. Berlin: Springer; 1995:945-946

51. M Kobayashi, T Fujino, T Kaneko, et al. Computer-Aided Surgery in Plastic and Reconstructive Surgery Using Laser Lithography Models and Virtual Reality Technique. Computer Aided Radiology. Berlin: Springer; 1995:1061-1065

52. Kobayashi M, Fujino T, Kaneko T, Yazawa M, Chiyokura H. Surgical simulation using a sterolithographic 3-dimensional replica. Computer Aided Radiology. Amsterdam, The Netherlands: Elsevier; 1998:551-554

53. Fujino T. 3D imaging in plastic simulations surgery. Computer Aided Radiology. Amsterdam, The Netherlands: Elsevier; 1998:564-568

54. Teschner M, Girod S, Girod B. Efficient and robust soft tissue prediction in craniofacial surgery simulation using individual patient's data sets. Computer Aided Radiology. Amsterdam, The Netherlands: Elsevier; 1999:635-639

55. Lo LJ, Marsh JL, Vikas PV, Vannier M. Craniofacial surgical simulation and outcome validation. Computer Aided Radiology. Berlin, Germany: Springer; 1995:789-794

56. Tanaka T, Kobayashi M, Fujino T, Chiyokura H. Simulation for facial lip expression using the facial muscle model. Computer Aided Radiology. Berlin, Germany: Springer; 1995: 878-881

57. Bockholt U, Mlynski G, Muller WK, G V. Using simulation of the airflow in the human nose for rhiosurgical therapy planning. Computer Aided Radiology. Amsterdam, The Netherlands: Elsevier; 1999:790-794

58. Steiner CP, LaPreston E, Hahn JF. Surgical navigation using preoperative and intraoperative imaging devices. Proceedings of Surgically Assisted Systems. Bellingham, WA: SPIH Optical Engineering Press; 1998:225-228

59. Cutting C, Grayson B, McCarthy JG, et al. A virtual reality system for bone fragment positioning in multisegment craniofacial surgical procedures. Plast Reconstr Surg 1998;102:2436-2443

60. Santler G. The computerized 3D-model surgery simulator-introduction of a new system. Computer Aided Radiology. Amsterdam, The Netherlands: Elsevier; 1999:908-912

61. Edwards P, Hawkes D, Hill D, et al. Augmentation of reality using an operating microscope for otolaryngology and neurosurgical guidance. J Image Guided Surg 1995;1:172-178

62. Kozarek RA. Virtual reality and gastrointestinal endoscopy, or, is virtual vision a speed bump on the road to telepresence? [editorial]. J Clin Gastroent 1997;24:130-132 
63. Burghart C, Hassfeld S, Raczkowsky J, Worn H, Rembold U. A surgical robotic system for maxillofacial surgery. Computer Aided Radiology. Amsterdam, The Netherlands: Elsevier; 1998:569-574

64. Satava RM. Medical applications of virtual reality. J Med Syst 1995;19:275-280

65. Bowersox J, Shah A, Jensen J, Hill J, Cordts P, Green P. Vascular applications of telepresence surgery: initial feasibility studies in swine. J Vasc Surg 1996;23:281-287

66. Bowersox J, Cornum R. Remote operative urology using a surgical telemanipulator system: preliminary observations. Urology 1998;52:17-22

67. Skari T. The cutting edge: heart surgery enters the age of robotics. Life 1998; Fall: 14-23

68. Stephenson ER, Sankholkar S, Ducko CT, Damiano RJ. Robotically assisted microsurgery for endoscopic coronary artery bypass grafting. Ann Thorac Surgery 1998;66:1064-1067

69. Shennib H, Bastawisy A, Mack M, Moll F. Computer-assisted telemanipulation: an enabling technology for endoscopic coronary artery bypass. Ann Thorac Surg. 1998;66:1060-1063

70. Satava RM. Cybersurgery: advanced technologies for surgical practice. Protocols in General Surgery. New York: Wiley-Liss; 1998

71. McGovern K. Outside the OR: The Future of surgical training.

72. Rodney WM. Will virtual reality simulators end the credentialing arms race in gastrointestinal endoscopy or the need for family physician faculty with endoscopic skills? [editorial; comment]. J Amer Board Fam Prac 1998;11:492-496

73. Waterworth JA. Virtual Reality in Medicine: A Survey of the State of the Art. UMEA, Sweden: Umea University; 1998:1-2

74. Longerich U, Carls F, Sailer H. The accuracy of the virtual patient system for craniomaxillofacial navigation. Computer Aided Radiology and Surgery. Amsterdam, The Netherlands: Elsevier Science; 1997:729-732

75. Schaich M, Bode A, Schwaderer E, Hoffman J, Reinert S, Claussen CD. Representation of individual anatomical relations using a virtual reality environment. Computer Assisted Radiology. Amsterdam, The Netherlands: Elsevier Science; 1999:631-634

76. Freysinger W. A Full 3D-navigation system in a suitcase. Computer Aided Radiology. Amsterdam, The Netherlands: Elsevier Science; 1999:640-644

77. WP Geis, HD Kim, PC McAfee, JG Kang, Brennan E. Synergistic benefits of combined technologies in complex, minimally invasive surgical procedures. Surg Endosc 1996;10:1025-1028

78. Smith DJ, Jr. Resident education-a casualty of managed care? [editorial]. Ann Plast Surg 1997;39:330-331 